\title{
Ziegen sind auch nur Menschen
}

\section{Zur Messung von kommunikativem Verhalten}

\section{Spitzer, Ulm}

Vor nicht allzu langer Zeit war an dieser Stelle die Rede davon, dass Hunde im Laufe ihrer Domestizierung die Eigenschaft angenommen haben, auf den Blick des Menschen mit Empathie anstatt mit Angst $\mathrm{zu}$ reagieren: Über Jahrtausende hinweg wählte der Mensch aus den ihn begleitenden Wölfen Tiere aus, die sich in seine Gemeinschaft einfügten, soziale Kompetenzen hatten, ihn anschauten und ,verstanden", was er von ihnen wollte (11). So entstand der Hund als eine neue Art aufgrund des Selektionsdrucks, den der Mensch implementierte: Sozialverhalten. Hunde schauen ihr Herrchen an, wenn dieses den Hund anschaut - sie erwidern den Blick; Wölfe tun dies nicht (6).

Der gemeinsame Vorfahre von Hund und Wolf ist längst ausgestorben, und Hunde sind mittlerweile auch dort verbreitet, wo es früher gar keine Wölfe gab ${ }^{1}$ - so erfolgreich waren sie als Gefährten des Menschen (4). Nach neuesten Erkenntnissen entstanden sie sogar zweimal unabhängig voneinander, zum einen in Ostasien und zum zweiten in Europa und angrenzenden westlichen Asien (3). Später kam es dann zu einer Durchmischung der Gene beider Arten, weil aus dem Osten kommende Menschen im Verlauf von Wanderungen und Kriegen ihre Hunde mitbrachten. Dies ist nicht ungewöhnlich, denn auch andere über Jahrtausende vom Menschen gezüchtete Arten, z. B. Weintrauben (8) oder Schafe (1), wurden erst domesti-

1 Die Ausbreitung von Hunden über mittlerweile den gesamten Globus folgte letztlich der Ausbreitung der bäuerlichen Lebensweise. Die „letzten Wiesen“ waren Südamerika und Südafrika, wo es Hunde erst seit weniger als 1400 Jahren gibt (4).

Nervenheilkunde 2016; 35: 709-710

Korrespondenzadresse

Prof. Dr. Dr. Manfred Spitzer

Universitätsklinikum Ulm

Klinik für Psychiatrie und Psychotherapie III

Leimgrubenweg 12, $89075 \mathrm{Ulm}$ ziert und verbreiteten sich dann über den Globus, wodurch es zu einer erneuten genetischen Durchmischung - auch mit den „wilden Vorfahren“ - kam.

Hunde schauen ihr Herrchen an, wenn dieses den Hund anschaut sie erwidern den Blick; Wölfe tun dies nicht.

Zurück zum Sozialverhalten: Nach ganz neuen Erkenntnissen haben nicht nur Hunde entsprechende Fähigkeiten, sondern Pferde (Equus caballus) auch (5). Dies verwundert nicht weiter, denn auch Pferde sind seit Jahrtausenden Begleiter des Menschen und wurden - ähnlich wie Hunde nicht nur nach bestimmten „brauchbaren“ Fähigkeiten ausgesucht und gezüchtet, sondern vor allem im Hinblick auf ihr Sozialverhalten. Bei Katzen hingegen scheint dies nie der Fall gewesen zu sein. Entsprechend schneiden sie auch schlecht in Tests ab, die messen, ob sie den Blick eines Menschen erwidern (7).

Was ist es eigentlich, das dieses Verhalten so bedeutsam macht? - Man braucht nicht lange nachzudenken: Es ist die erfolgreiche Kommunikation zwischen zwei „Partnern“ zur Lösung eines Problems oder einer Aufgabe. Um die hierfür notwendigen Fähigkeiten zu untersuchen, ohne den betreffenden „Partner“ fragen zu müssen bzw. zu können, wurden sowohl in der Entwicklungspsychologie („Säuglingsforschung") als auch in der Tierpsychologie Methoden entwickelt, die allein auf Verhaltensbeobachtung in bestimmten standardisierten sozialen Situationen beruhen. Nur so kann man herausbekommen, welche Fähigkeiten es genau sind, die bei erfolgreicher Kommunikation eine Rolle spielen.

Nun kann man den Standpunkt vertreten, dass gerade aufgrund der Selektion von Hunden und Pferden durch den Menschen im Rahmen ihrer Jahrtausende langen Züchtung zur Zusammenarbeit beim Jagen oder Ackerbau auch die menschliche
Fähigkeit zum Zeigen und Verstehen eines Zeigevorgangs (es geht nicht um die Spitze des Zeigefingers oder der Nase, sondern um das, worauf diese "Pfeile“ deuten) irgendwie auf die andere Art übergegangen ist. Anders gefragt: Gibt es auch ein Verständnis von Intentionalität bei Tieren, die nicht auf die Zusammenarbeit mit Menschen über Jahrtausende hin "getrimmt" worden waren, z. B. bei Ziegen? Diese wurden zwar auch gezüchtet, aber vor allem wegen ihrer Milch und ihres Fleischs (neben Häuten für Trinksäcke und Mohair für warme Kleidung).

Daher ist es umso bemerkenswerter, dass britische Wissenschaftler von der Queen Mary University of London untersucht haben, ob auch bei Ziegen das bei Hunden und Pferden nachgewiesene Kommunikationsverhalten vorkommt. In ihrer schönen Arbeit mit dem Titel „Ziegen zeigen publikumsabhängiges, auf Menschen gerichtetes Blickverhalten in einer Problemlöseaufgabe" (9, Übersetzung durch den Autor) wurden 34 Ziegen - 17 Weibchen und 17 kastrierte Männchen im Alter von 2 bis 15 Jahren - einzeln immer der gleichen Testprozedur unterzogen, in der die Tiere, mit einem unlösbaren Problem konfrontiert waren. Alle Tiere waren sehr an ihre Tierpfleger gewöhnt und wurden in der Testsituation mit einem Menschen konfrontiert, der entweder vorwärts und zu ihnen schaute, oder der ihnen den Rücken zukehrte und wegschaute ( $\triangleright$ Abb.).

Die Autoren gingen hierbei wie folgt vor: Zunächst lernte die Ziege, den Deckel eines durchsichtigen Gefäßes abzunehmen, um an leckeres Futter heranzukommen. Drehte man dann das Gefäß um, hatte die Ziege mehr Mühe, das Gefäß umzuschubsen, um an das Futter heranzukommen. Schließlich befestigte man den Deckel am Gefäß, sodass die Ziege das Futter zwar sehen, aber nicht mehr an das Futter herankommen konnte. Bei diesen Vorbereitungsdurchgängen wurden zwei Ziegen ausgeschlossen, die die Sache offensichtlich 

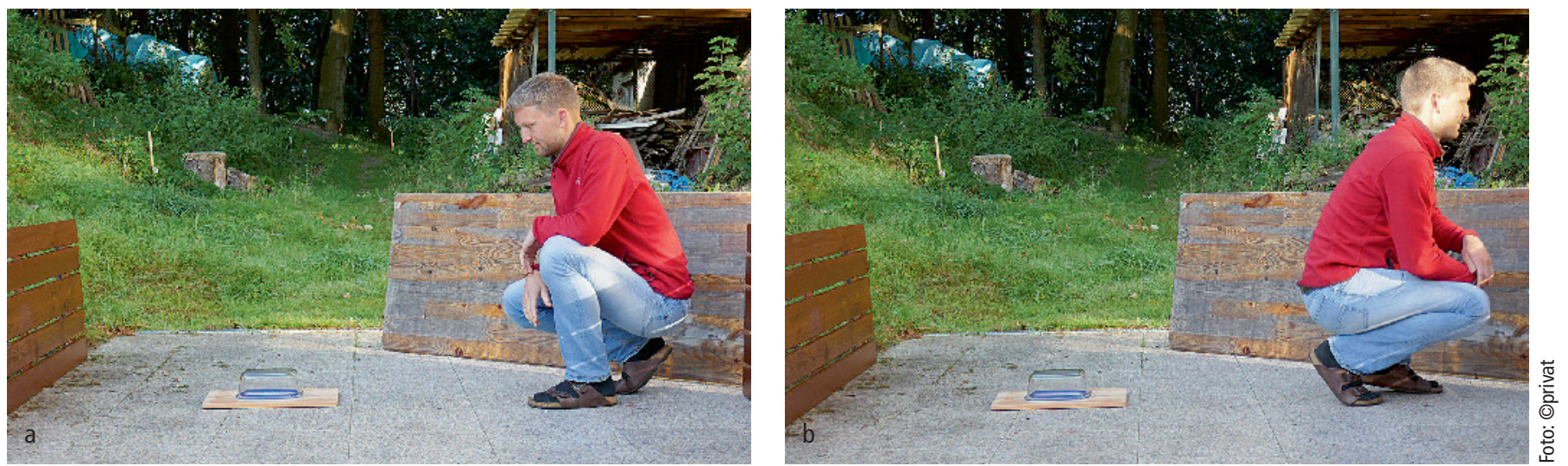

Abb. Umgedrehtes Gefäß mit Deckel (Problem) und anwesender (dem Tier gut bekannter) Mensch, der sich hinwendet (links) oder sich abwendet (rechts) zum Test des kommunikativen Verhaltens von Ziegen (nach 9, Fig. 1 a, b).

nicht gut verstanden hatten. Die anderen 32 Ziegen (15 weiblich) wurden dem eigentlichen Test mit nur einem zweiminütigen Durchgang und zwei Bedingungen per Zufall zugeteilt (16 Ziegen pro Bedingung): Der neben dem Gefäß befindliche Pfleger war entweder dem Gefäß zugewandt oder abgewandt. Ein zweiter Pfleger (in der Abbildung nicht zu sehen) befand sich etwa 2,5 Meter entfernt und diente dazu, die Neigung der Tiere, überhaupt einen Menschen anzuschauen, während des Versuchs (als Kontrolle) zu messen.

Das Verhalten der Tiere in diesem Test wurde per Video aufgezeichnet und mittels standardisierter Software von zwei unabhängig voneinander arbeitenden Personen ausgewertet (wobei sich eine sehr gute Übereinstimmung zeigte). Ausgewertet wurde u. a., ob und wie lange sich die Ziege einem anwesenden Menschen zuwandte und ihn anschaute. Wie oft dies geschah, und ob die Ziege abwechselnd auf den Menschen und dann wieder das Gefäß blickte, sich also gewissermaßen an einen anwesenden Menschen mit der durch ihr Blickverhalten kommunizierten „Bitte“ wandte, ihr doch bei der für sie allein unlösbaren Aufgabe zu helfen. Wie bereits erwähnt, tun dies Hunde und Pferde auch.

Was also taten die Ziegen? Beim Training wurden sie zunächst immer besser im Lösen des (für sie lösbaren) Problems. Im ersten Durchgang brauchten sie hierfür im Mittel 9,2 Sekunden, im zweiten Durchgang 6,2 Sekunden und im dritten 5,4 Sekunden. Keine der Ziegen schaute dabei zum anwesenden Menschen. Im Testdurchgang schauten die Ziegen den ihnen zugewandten Menschen signifikant früher $(\mathrm{p}<0,001)$, länger $(\mathrm{p}=0,02)$ und häufiger $(\mathrm{p}=0,013)$ an als den ihnen abgewandten. Auch die Zeit bis zum ersten Hin- und Herblicken zwischen Futter und Mensch war beim zugewandten Menschen signifikant geringer $(p=0,038)$ sowie die Anzahl dieser Blickwendungen signifikant größer ( $p=0,033$ ). Für den zweiten als Kontrolle weiter entfernt stehenden Menschen waren keine signifikanten Unterschiede zwischen beiden Gruppen festzustellen.

Die Ziegen verhielten sich in diesem Test damit insgesamt nicht anders als von anderen Autoren bereits für Hunde und Pferde beschrieben. Damit kann bezweifelt werden, dass die Domestizierung von Tieren nur dann besseres kommunikatives Verhalten bewirkt, wenn speziell für solches Verhalten selektiert wird (wie im Falle von Hunden und Pferden anzunehmen ist). Vielmehr scheint eine verbesserte Kommunikation mit Menschen ganz allgemein zu resultieren, wenn Domestizierung überhaupt erfolgt. „Thus, domestication has probably had a much broader impact on heterospecific communication than previously believed“, kommentieren die Autoren ihre Ergebnisse (9).

\section{Eine verbesserte Kommunikation} mit Menschen scheint ganz allgemein zu resultieren, wenn Domestizierung überhaupt erfolgt.

Menschen kommunizieren, sehr viel und vor allem miteinander. Sie lösen dadurch Probleme effektiv und schnell. Offensichtlich färbte dies ab auf andere Arten, die eng mit dem Menschen zusammenlebten. Für dieses „Abfärben“ genügt offenbar geteilter Lebensraum und Lebensvollzug; geteilte Arbeit muss es nicht unbedingt sein.

\section{Literatur}

1. Chessa B et al. Revealing the history of sheep domestication using retrovirus integrations. Science 2009; 324: 532-536.

2. Douglas C. Goats, sheep and cows could challenge dogs for title of 'man's best friend'. The Conversation, 8.7.2016 (https://theconversation.com/ goats-sheep-and-cows-could-challenge-dogs-fortitle-of-mans-best-friend-62206.

3. Frantz LAF et al. Genomic and archaeological evidence suggest a dual origin of domestic dogs. Science 2016; 352: 1228-1231.

4. Larson et al. Rethinking dog domestication by integrating genetics, archeology, and biogeography. PNAS 2012; 109: 8878-8883.

5. Malavasi R, Huber L. Evidence of heterospecific referential communication from domestic horses (Equus caballus) to humans. Animal Cognition 2016; 19: 899-909.

6. Miklosi A et al. A simple reason for a big difference: wolves do not look back at humans, but dogs do. Curr Biol 2003; 13: 763-766.

7. Miklosi A et al. A comparative study of the use of visual communicative signals in interactions between dogs (Canis familiaris) and humans and cats (Felis catus) and humans. J Comp Psychol 2005; 119: 179-186.

8. Myles $\mathrm{S}$ et al. Genetic structure and domestication history of the grape. PNAS 2011; 108: 3530-3535.

9. Nawroth C, Brett JM, McElligott AG. Goats display audience-dependent human-directed gazing behaviour in a problem-solving task. Biol Lett 2016; 12: 20160283.

10. Savalli C, Ades C, Gaunet F. Are dogs able to communicate with their owners about a desirable food in a referential and intentional way? PLoS ONE 2014; 9(9): e108003.

11. Spitzer M. Hunde sind auch nur Menschen. Nervenheilkunde 2015; 34: 755-757. 